\title{
A STUDY TO DETERMINE MATERNAL AND FETAL OUTCOME IN HIGH RISK PREGNANCIES
}

\section{DR.MEENAL PATVEKAR ${ }^{1}$, DR.BHARGAVI ${ }^{2}$, DR.PRAHARAJU RAJITHA NIDHI ${ }^{3} \&$ DR.AVISHA MALU $^{4}$}

${ }^{I} M D$ (Prof and HOU of Obs and Gyn) Dr. D Y Patil Medical College, Pune, India

${ }^{2} \mathrm{MS}$ ( Obs and Gyn),3 rd year pg resident, Dr.D Y Patil Medical College, Pune, India

${ }^{3} \mathrm{MS}$ (Obs and Gyn),2 nd year pg resident,Dr.D Y Patil Medical College,Pune,India

${ }^{4} \mathrm{MS}$ (Obs and Gyn), 1 st year pg resident ,Dr.D Y Patil Medical college ,Pune,India

\begin{abstract}
Background: High risk pregnancy is any pregnancy in which there is a maternal or fetal factor that may adversely affect the outcome. In India, about 20-30 \% pregnancies belong to high risk category, which is responsible for $75 \%$ of perinatal morbidity and mortality. In order to improve the outcome of a high risk pregnancy, we must identify high risk factors and attempt to mitigate care to all these patients with high risk factors.

Methods: The present study is an observational study focusing on maternal and fetal outcome in high risk pregnancies in a tertiary centre over 2 years, conducted in Dr DY Patil Medical College, Hospital and Research Centre, Pimpri, Pune. A pre designed semi structured, patient friendly questionnaire was prepared based on review of literature on high risk pregnancies.

Results: In our study it was observed that patients of 20-25 years and 26-30 years were in reproductive age group and accounted for majority of the cases i.e. $42.1 \%$ and $36.9 \%$ respectively. A total of 1264 patients were admitted to our hospital between 34 to 40 weeks of gestational age, of which 384 patients were recognized as to have a high risk factors. The incidence of high risk pregnancy in our study was $30.37 \%$. In our study, severe anaemia was the leading high risk factor, accounting for $23.4 \%$.These patients were those with haemoglobin $<7 \mathrm{~g} / \mathrm{dl}$ who required blood transfusion. Maternal outcome was the mainstay of our study. Anaemia being the single largest high risk factor, needed correction and therefore the use of parenteral iron and blood transfusion together accounted for $61.35 \%$ of patients. Blood transfusion was given to patients who were severe anaemic i.e. $\mathrm{Hb}$ was $<7 \mathrm{~g} / \mathrm{dl}$, which accounted for $23.2 \%$. This was followed by other morbid end organ disease like hypertension in pregnancy (16.1\%), preterm labour (13.5\%), previous LSCS (10.4\%).Among the maternal outcome measures Post-partum haemorrhage accounted for $11.4 \%$ in our study which was a dangerous morbid factor anytime in obstetrics. Wound infection accounted for $10.5 \%$ among woman who underwent LSCS in our study, of these $5.2 \%$ required secondary resuturing for both LSCS and episiotomy wound gape. Sudden blindness and acute renal failure were one of the end organ damage was seen in $1.75 \%$ of patients who had hypertension and severe antepartum haemorrhage. Amongst the fetal outcome,our study showed, preterm births which was $12.9 \%$ followed by preterm with IUGR i.e. $11.9 \%$ and severely growth restricted babies accounted for $8.9 \%$.The commonest cause for preterm labour, preterm with IUGR and IUGR babies was maternal anaemia and hypertension. Our study showed $45.2 \%$ of low birth weight babies which was significantly associated with high risk pregnancy. Of the total 402 babies born in our study, 258 babies
\end{abstract}




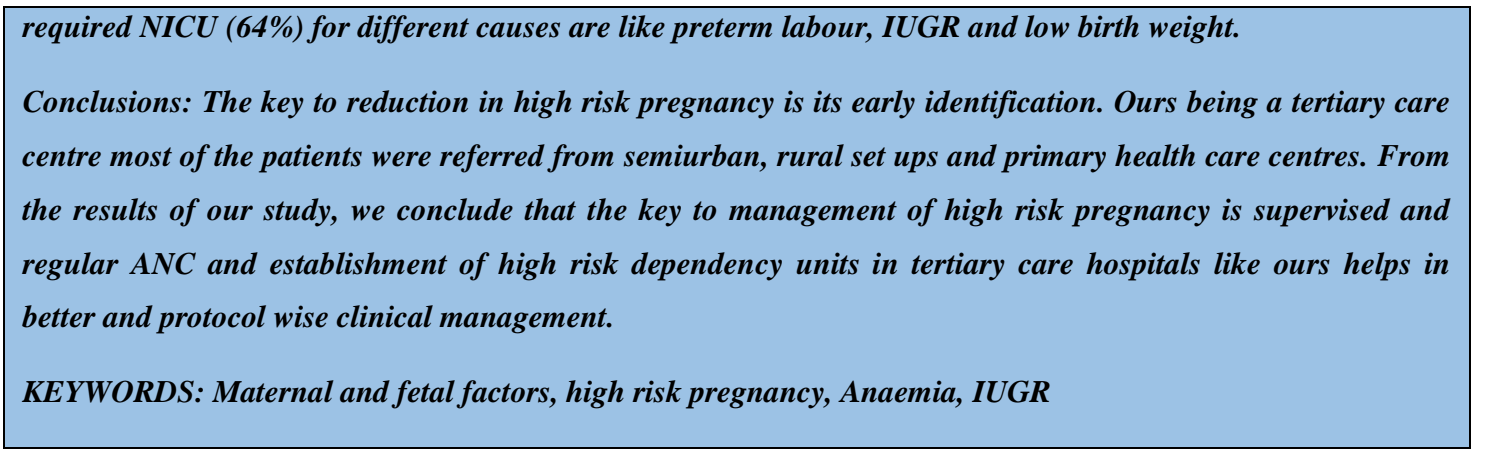

Received: Dec 23, 2020; Accepted: Jan 13, 2021; Published: Feb 02, 2021; Paper Id.: IJMPSJUN20211

\section{INTRODUCTION}

High risk pregnancy is any pregnancy in which there is a maternal or fetal factor that may adversely affect the outcome. High risk pregnancies are defined as a situation in which mother or foetus or neonate is at a high risk of mortality or morbidity ${ }^{(1)}$.These are those mothers who either suffer from complications embarking on pregnancy or encounter complications during pregnancy and during labour.

In India, about 20-30 \% pregnancies belong to high risk category, which is responsible for $75 \%$ of perinatal morbidity and mortality.

A few to mention high risk conditions include ; medical conditions like anaemia, hypertension in pregnancy, thrombocytopenia ,coagulation disorders in pregnancy, Gestational diabetes mellitus, hyperthyroidism, cardiovascular diseases in pregnancy, obesity (BMI $>30 \mathrm{~kg} / \mathrm{m}^{2}$ ), HIV/AIDS during pregnancy and other conditions like antepartum haemorrhage, IUGR, previous LSCS, preterm labour and extremes of age.

\section{AIMS AND OBJECTIVES}

- To study maternal and fetal outcome in high risk pregnancies

- To study the incidence of high risk pregnancies in a tertiary care hospital.

\section{MATERIAL AND METHODS}

The present study is an observational study focusing on maternal and fetal outcome in high risk pregnancies in a tertiary centre, conducted on384 patients of dept. of Obstetrics and Gynaecology of Dr. D.Y. Patil Medical College, Hospital and Research Centre, Pimpri, Pune. The period of study was from August 2018 to July 2020. Apredesigned semi- structured, patient friendly questionnaire was prepared based on review of literature on high risk pregnancies.

\section{Inclusion Criteria:}

- Gestational age more than 34 weeks

- Patients with high risk factors ( high risk pregnancies )

\section{Exclusion Criteria:}

- Less than 34 weeks

- Patients without high risk factors pregnancy 


\section{Source of Funding}

- Investigations done on OPD basis are all routinely done in 34-40 weeks gestational age patients and the cost will be borne by the patient.

- The cost of investigations for IPD patients is nil and free of cost in the institute.

- The consent of workup required will be self -funded.

Results:The present study was conducted with aim to study maternal and fetal outcome in high risk pregnancies and to study the incidence of high risk pregnancies in tertiary care hospital. Total of 384 study subjects were enrolled.

Table 1: Age wise distribution in high risk pregnancy

\begin{tabular}{|l|l|l|}
\hline $\begin{array}{l}\text { Age } \\
\text { (years) }\end{array}$ & $\begin{array}{l}\text { No of patients } \\
(\mathbf{n = 3 8 4})\end{array}$ & $\begin{array}{l}\text { Percentage } \\
(\%)\end{array}$ \\
\hline Up to 19 & 46 & $11.9 \%$ \\
\hline $20-25$ & 162 & $42.1 \%$ \\
\hline $26-30$ & 142 & $36.9 \%$ \\
\hline $31-35$ & 34 & $8.8 \%$ \\
\hline$>35$ & 0 & $0 \%$ \\
\hline
\end{tabular}

In our study, patients of 20- 25 years and 26-30 years were in reproductive age group and accounted for majority of the cases i.e. $42.1 \%$ and $36.9 \%$ respectively.

\section{Histogram showing the incidence of high risk cases}

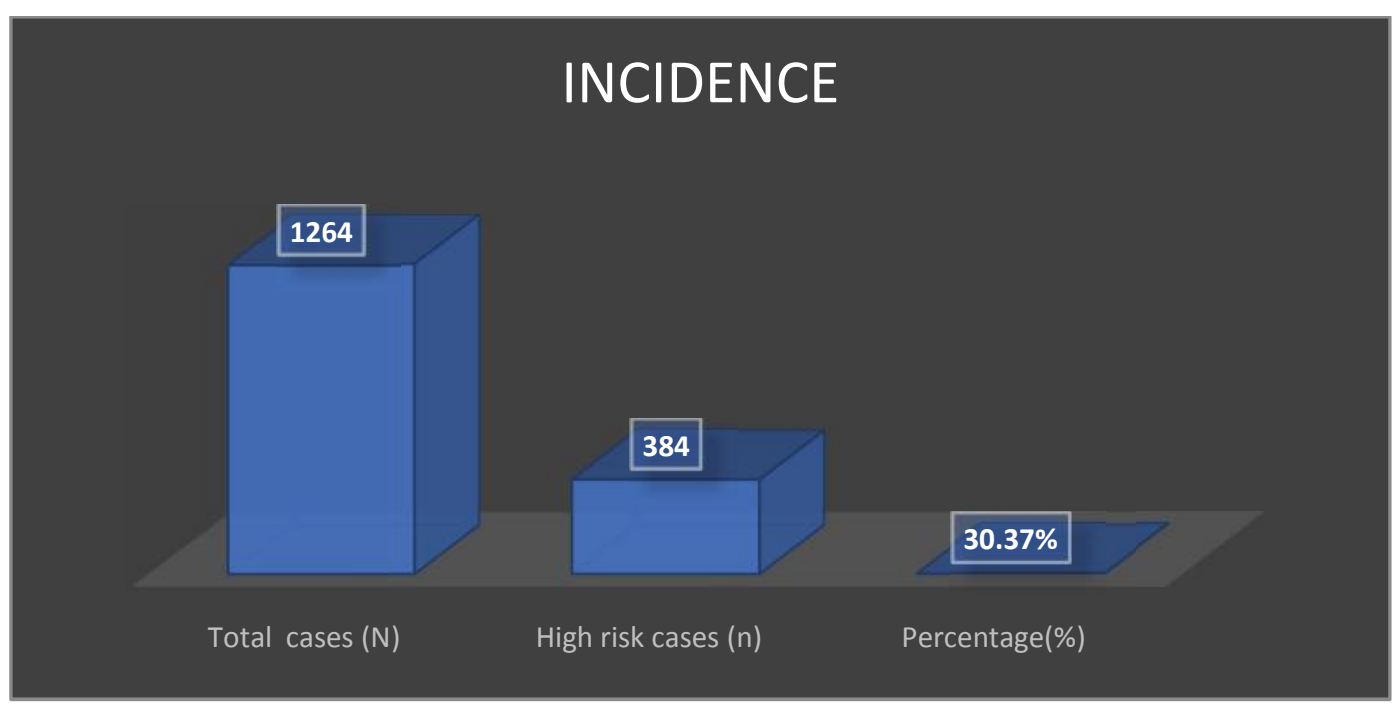

A total of 1264 patients were admitted to our hospital between 34 to 40 weeks of gestational age, of which 384 patients were recognized as to have a high risk factors accounting up to $30.37 \%$. 
Table 2: High risk factors wise distribution in high risk pregnancy

\begin{tabular}{|l|l|l|}
\hline \multicolumn{1}{|c|}{ High risk factors } & \multicolumn{1}{|c|}{$\begin{array}{c}\text { Number of } \\
\text { patients } \\
(\mathbf{n = 3 8 4 )}\end{array}$} & \multicolumn{1}{c|}{$\begin{array}{c}\text { Percentage } \\
\%\end{array}$} \\
\hline Severe Anaemia & 90 & $23.4 \%$ \\
\hline Hypertension in pregnancy & 62 & $16.14 \%$ \\
\hline Preterm labour & 52 & $13.54 \%$ \\
\hline Previous LSCS & 40 & $10.4 \%$ \\
\hline IUGR & 36 & $9.3 \%$ \\
\hline Antepartum haemorrhage & 28 & $7.29 \%$ \\
\hline Gestational diabetes mellitus & 24 & $6.25 \%$ \\
\hline Breech presentation & 20 & $5.2 \%$ \\
\hline Twin gestation & 18 & $4.68 \%$ \\
\hline Obesity & 12 & $3.12 \%$ \\
\hline Coagulation disorders & 2 & $0.52 \%$ \\
\hline
\end{tabular}

Table 2; shows high risk factors wise distribution in high risk pregnancy.

In our study, severeanaemia was the leading high risk factor, accounting for $23.4 \%$.These patients werethose with haemoglobin $<7 \mathrm{~g} / \mathrm{dl}$.This was followed by hypertension in pregnancy (16.1\%), preterm labour (13.5\%), previous $\operatorname{LSCS}(10.4 \%)$.

\section{Single/multiple high risk factors}

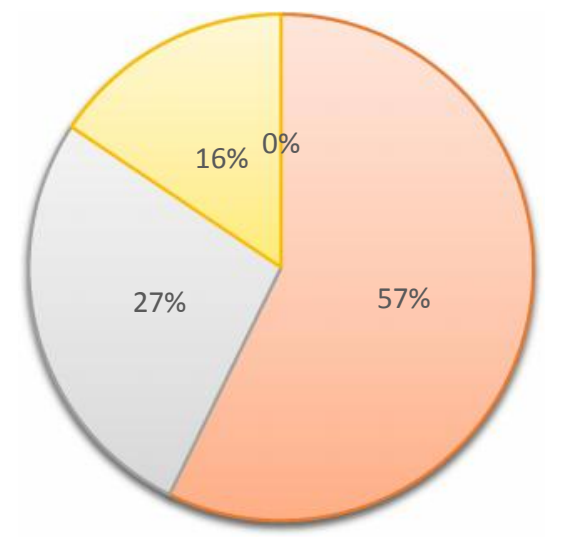

$\square$ High risk factors $\square 1$ high risk factor $\square \geq 2$ high risk factors $\square \geq 3$ high risk factors

Pie chart showing single/multiple high risk factors wise distribution in high risk pregnancy

High risk factors may be single / multiple in any given patient. Our study shows that single high risk factor accounted $57.2 \%$ of patients. Patients with two and more high risk factors were $27 \%$ and three and more high risk factors were $15.6 \%$. 
Table 3: Maternal outcome (Morbidity) wise distribution in high risk pregnancy

\begin{tabular}{|l|l|l|}
\hline \multicolumn{1}{|c|}{ Maternal Complications } & \multicolumn{1}{|c|}{$\begin{array}{c}\text { Total No. of } \\
\text { patients(n=228) }\end{array}$} & \multicolumn{1}{|c|}{$\begin{array}{c}\text { Percentage } \\
(\%)\end{array}$} \\
\hline Parenteral Iron & 87 & $38.1 \%$ \\
\hline Blood transfusion & 53 & $23.2 \%$ \\
\hline PPH & 26 & $11.4 \%$ \\
\hline Wound infection & 24 & $10.5 \%$ \\
\hline Febrile morbidity & 18 & $7.8 \%$ \\
\hline Secondary resuturing & 12 & $5.2 \%$ \\
\hline SICU admission & 4 & $1.75 \%$ \\
\hline End organ damage & 4 & $1.75 \%$ \\
\hline
\end{tabular}

Table 3 shows maternal outcome wise distribution in high risk pregnancy.

This is the mainstay of our study. The above table shows how high risk pregnancy impacts on the maternal outcome. Anaemia being the single largest high risk factor, needed correction and therefore the use of parenteral iron and blood transfusion together accounted for $61.3 \%$ of patients. Blood transfusion was given to patients who were severe anaemici.e. $\mathrm{Hb}$ was $<7 \mathrm{~g} / \mathrm{dl}$, which accounted for $23.2 \%$. Haemorrhage and wound infection followed with $11.4 \%$ and $10.5 \%$ respectively. The worst insult was end organ damage which was $1.75 \%$ and included hypertension induced sudden blindness and acute renal failure in patient with severe antepartum haemorrhage.

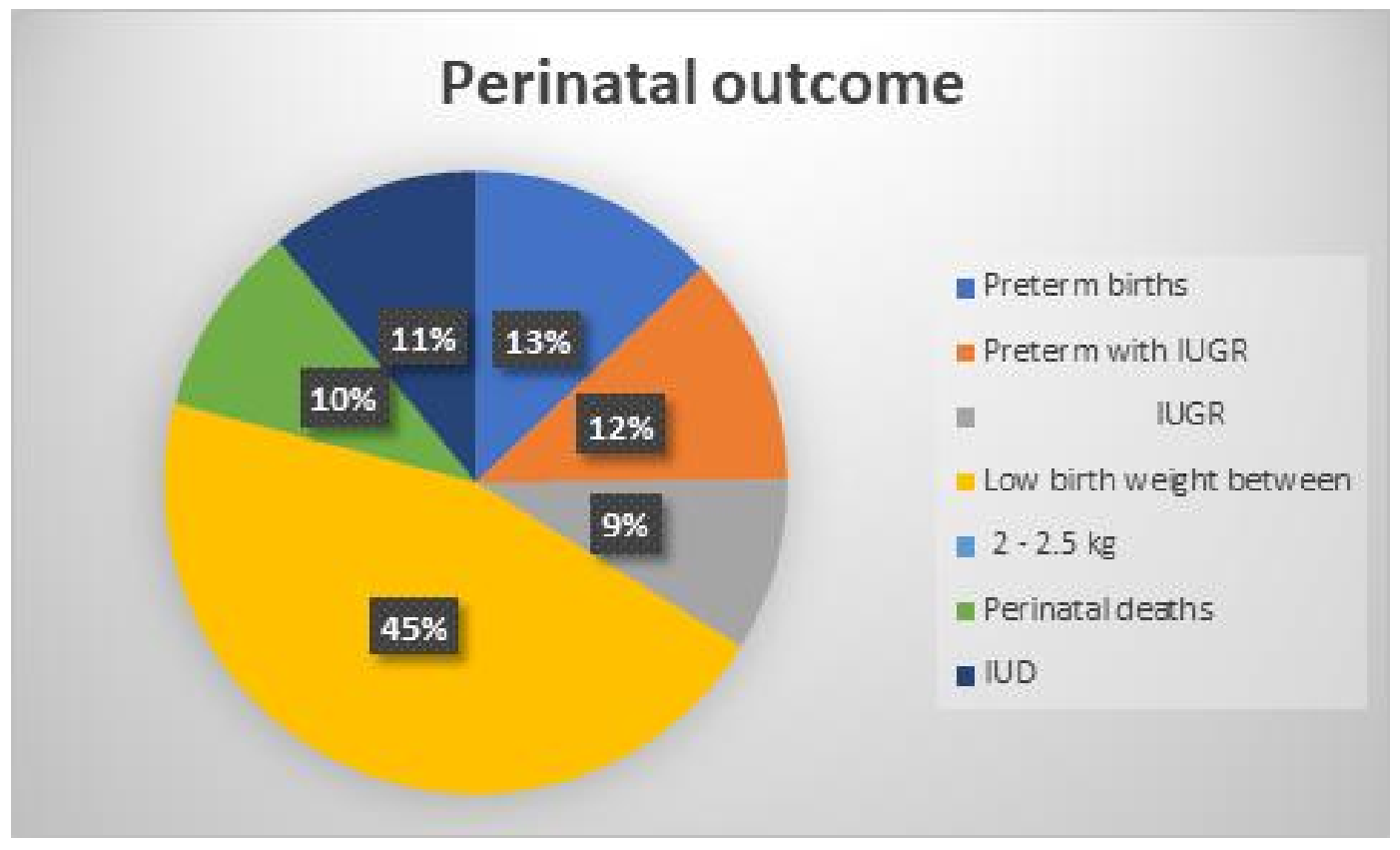

Pie chart shows neonatal outcome distribution in high risk pregnancy

The other important aspect of our study was fetal outcome. Our study showed, preterm births which was $12.9 \%$ followed by preterm with IUGR i.e. $11.9 \%$ and IUGR accounted for $8.9 \%$.Maternal anaemia was the foremost cause of preterm labour and IUGR babies. In addition to anaemia as a major risk factor, Low birth weight babies who accounted up 
to $45.2 \%$ and hypertension emerged as cause for these babies. One of the worst outcomes in this study was perinatal deaths which was $9.9 \%$. The cause of these perinatal deaths was preterm labour which was 52\%, antepartumhaemorrhage and severe preeclampsia.

Table 4: NICU requirements wise distribution with live births in high risk pregnancy

\begin{tabular}{|l|l|l|}
\hline \multicolumn{1}{|c|}{ NICU parameters } & \multicolumn{1}{|c|}{$\begin{array}{c}\text { No of neonates } \\
(\mathbf{n = 2 5 8})\end{array}$} & \multicolumn{1}{|c|}{$\begin{array}{c}\text { Percentage } \\
(\%)\end{array}$} \\
\hline $\begin{array}{l}\text { NICU admission (observation, needing 02,nasal } \\
\text { prongs) }\end{array}$ & 186 & $72 \%$ \\
\hline NICU admission needing ventilator & 50 & $19.3 \%$ \\
\hline NICU admission needing CPAP & 22 & $8.5 \%$ \\
\hline
\end{tabular}

Table 4; shows NICU requirements wise distribution with live births in high risk pregnancy.

Of the total 402 babies born in our study,258 babies required NICU (64\%) which showed babies of these mothers having high risk factors. This included either only observation/requiring 02 or on nasal prongs. The reasons for this would be APGAR score < 7 , transient tachypnoea/perinatal hypoxia and respiratory distress and is seen in mothers with severe anaemia, preterm labourand PROM.

\section{DISCUSSIONS}

In our study, patients of 20- 25 years and 26-30 years were in reproductive age group and accounted for majority of the cases i.e. $42.1 \%$ and $36.9 \%$ respectively. Reproductive outcome during this age is good and associated with lower maternal and fetal complications. A total of 1264 patients were admitted to our hospital between 34 to 40 weeks of gestational age, of which 384 patients were recognized as to have a high risk factors accounting up to $30.37 \%$.Vasavi Kolluru et al ${ }^{(2)}$ conducted a prospective study on 200 pregnant woman with term gestation, the incidence of high risk pregnancy was found to be $20 \%$ as compared to our study. In our study, severe anaemia was the leading high risk factor, accounting for $23.4 \%$ .These patients were those with haemoglobin $<7 \mathrm{~g} / \mathrm{dl}$ who required blood transfusion .This was followed by hypertension in pregnancy (16.1\%), preterm labour (13.5\%), previous LSCS (10.4\%).Patel A et al ${ }^{(3)}$, conducted a prospective observational cohort study in 72750 woman, to assess the trend in the prevalence of anaemia and its outcome on pregnancy. Over $90 \%$ of the women included in the study were anaemic and hence concluded, maternal anaemia was associated with enhanced risk of stillbirth, neonatal deaths and LBW. This study shows a significantly high percentage of anaemia. Kovvuru Ashrita et al ${ }^{(4)}$ conducted retrospective study on 1176 pregnant women; hypertension was the most emerging high risk factor in their study (53.1\%) as compared to our study which was (16.14\%). The next leading high risk factor in our study was preterm labour (13.5\%) as compared to Kovvuru Ashrita et al ${ }^{(4)}$ study in 1176 woman which showed $79 \%$ of preterm delivery. CA Iyoke ${ }^{(5)}$ et all conducted a prospective cohort studyin 870 woman ,the risk of caesarean section in previous caesarean section was found to be $75.8 \%$ compared to our study previous LSCS was 10.4\%.Post-partumhaemorrhage accounted for $11.4 \%$ in our study which was a dangerous morbid factor anytime in obstetrics, compared to Sam Ononge ${ }^{(6)}$ et al prospective cohort study among 1188 woman, incidence was $9 \%$. One of the 
worst outcomes in this study was perinatal deaths which was 9.9\%. The cause of these perinatal deaths was preterm labour which was $52 \%$, antepartumhaemorrhage and severe preeclampsia. Our study accounted for $72 \%$ of babies requiring NICU for observation due to perinatal hypoxia, requiring $\mathrm{O} 2$ in severe anaemia and on nasal prongs compared toC Dani et al ${ }^{(7)}$ study showed that out of 63,537 newborns, $1.1 \%$ were respiratory distress syndrome (RDS) and $0.9 \%$ were transient tachypnoea (TT) required NICU.

\section{CONCLUSIONS}

From the results of our study, we conclude that the key to management of high risk pregnancy is supervised and regular ANC. Outreach camps and high risk clinic establishment is the need to prevent morbidity and mortality in patients from rural areas. Clinical expertise and prompt treatment especially in patients of severe preeclampsia, eclampsia and postpartumhaemorrhage can prevent maternal and perinatal deaths. Establishment of high risk dependency units in tertiary care hospitals like ours helps in better and protocol wise clinical management.

\section{REFERENCES}

1. Moosazadeh M, Naghibi SA, Khosravi S, Afshari M, Afsargharehbagh R. Outcomes of High-Risk Pregnancies in Northern Iran: Multivariate Logistic Regression Model. Iranian Journal of Health Sciences. 2015; 3(4):40-6.

2. Manju Chaudhary \& Naseeb Kumar, "Effect of Socioeconomic Status and Cultural Factors with Various Unsuccessful Reproductive Outcomes", International Journal of Environment, Ecology, Family and Urban Studies (IJEEFUS), Vol. 6, Issue 1, pp. 131-134

3. Kolluru V, Reddy A. Study of high risk scoring in pregnancy and perinatal outcome. Indian J Obstet Gynecol Res. 2016; $3(4): 407-9$.

4. Patel A, Prakash AA, Das PK, Gupta S, Pusdekar YV, Hibberd PL. Maternal anaemia and underweight as determinants of pregnancy outcomes: cohort study in eastern rural Maharashtra, India. BMJ open. 2018 Aug 1; 8(8):e021623.

5. Kovvuru Ashrita, Kalvakunta Sravagnya. High risk pregnancies - The maternal and fetal outcomes in a tertiary care unit. IAIM, 2020; 7(6): 14-20.

6. Isawumi A.I, Akindele R.A. \& Fasanu A.O , "Low Dose Gonadotropin Protocol for Ovulation Induction in Low Resource Centre “, BEST: International Journal of Humanities, Arts, Medicine and Sciences (BEST: IJHAMS), Vol. 3, Issue 7, pp. 51-58

7. Iyoke CA, Ugwu GO, Ezugwu FO, Lawani OL, Onah HE. Risks associated with subsequent pregnancy after one caesarean section: A prospective cohort study in a Nigerian obstetric population. Nigerian Journal of Clinical Practice. 2014 Jun 23; $17(4): 442-8$.

8. Ononge S, Mirembe F, Wandabwa J, Campbell OM. Incidence and risk factors for postpartum haemorrhage in Uganda. Reproductive health. 2016 Dec; 13(1):1-7.

9. Meenal Agarwall, Chaula Doshi \& Vaishali Khot, "Emergency Cesarean Section in a Patient with Rheumatic Heart Disease with Gestational HTN \& Neurocysticercosis: An Anesthetic Challenge “, International Journal of General Medicine and Pharmacy (IJGMP), Vol. 4, Issue 1, pp. 89-92

10. Nugroho Susanto, Chatarina Uw, Hari Basuki N, Stefanus Supriyanto, Kuntoro, Lutfan Lazuardi, Windhu Purnomo, Florentina Sustini, "Utilization of Geo-spatial as Method for Trends Pregnancy Complication, a Case Study of Rural and Urban”, International Journal of Environment, Ecology, Family and Urban Studies (IJEEFUS), Vol. 5, Issue 2, pp. 21-30 
11. "Dani C, Reali MF, Bertini G, Wiechmann L, Spagnolo A, Tangucci M, Rubaltelli FF. Risk factors for the development of respiratory distress syndrome and transient tachypnoea in newborn infants. Italian Group of Neonatal Pneumology. European Respiratory Journal. 1999 Jul 1; 14(1):155-9. 